\title{
BMJ Open CoCo20 protocol: a pilot longitudinal follow-up study about the psychiatric outcomes in a paediatric population and their families during and after the stay- at-home related to coronavirus pandemic (COVID-19)
}

\author{
Morgane Gindt, ${ }^{1,2}$ Arnaud Fernandez (D) , ${ }^{1,2}$ Aurelien Richez, ${ }^{1,2}$ Ophelie Nachon, ${ }^{1,2}$ \\ Michele Battista, ${ }^{1,2}$ Florence Askenazy ${ }^{1,2}$
}

To cite: Gindt M, Fernandez A, Richez $\mathrm{A}$, et al. $\mathrm{CoC} 020$ protocol: a pilot longitudinal follow-up study about the psychiatric outcomes in a paediatric population and their families during and after the stay-at-home related to coronavirus pandemic (COVID-19). BMJ Open 2021;11:e044667. doi:10.1136/ bmjopen-2020-044667

- Prepublication history for this paper is available online. To view these files, please visit the journal online (http://dx.doi. org/10.1136/bmjopen-2020044667).

$M G$ and $A F$ are joint first authors.

Received 09 September 2020

Revised 11 March 2021

Accepted 15 March 2021

D Check for updates

(c) Author(s) (or their employer(s)) 2021. Re-use permitted under CC BY-NC. No commercial re-use. See rights and permissions. Published by BMJ.

For numbered affiliations see end of article.

Correspondence to Dr Arnaud Fernandez; fernandez.a@pediatriechulenval-nice.fr

\section{ABSTRACT}

Introduction In the context of a viral outbreak and the stay-at-home measures, a significant increase in psychological distress, such as stress or fear behaviours, has previously been reported in adult and paediatric population. Children and adolescents seem to be particularly at risk of developing psychiatric disorders during and after the stay-at-home but evidences are lacking. The main objective of this article is to present the methodology of Coronavirus Confinement 2020 (CoCo20) Study, which aims to assess the impact of the coronavirus pandemic (COVID-19) and stay-at-home on the development of psychiatric disorders, including post-traumatic stress disorder (PTSD), in children and adolescents.

Methods and analysis We describe a longitudinal and multicentre study in the paediatric population during and after stay-at-home related to COVID-19 pandemic. Inclusions started on 30 March 2020 for 6 months. This study is proposed to all consecutive paediatric outpatients consulting during and after stay-at-home related to COVID-19 pandemic in medical-psychological centres and in a paediatric psychotrauma centre and/or calling the emergency COVID-19 hotline. We perform standardised and internationally validated psychiatric assessments (Diagnosis Infant and Preschool Assessment, Kiddie Schedule for Affective Disorders and SchizophreniaPresent and Lifetime Version) together with anxiety, attention deficit hyperactivity disorder, PTSD, parenting stress and somatic symptoms scales during five visits (baseline, 1 week after baseline, 1 month after baseline, 1 week after the end of the containment and 1 month after the end of the containment) in patients and their families enrolled during the containment and during three visits in case of enrolment after the containment. The inclusion period will end in 30 November 2020.

Ethics and dissemination The protocol has been approved by the Ethics Committee of Cote d'Azur University « CERNI » (number 2020-59). All patients and their legal caregivers provide a written informed consent on enrolment in the study. We will submit the results
Strengths and limitations of this study

Team reactivity in the design of psychiatric protocol using standardised and internationally validated tools.

- Clinical research in child and adolescent psychiatry with longitudinal evaluations is needed to better understand the development of psychiatric disorder in a disaster context such as pandemic.

- Involve children and adolescents during the stayat-home is very challenging and at risk of limited sample size.

of the study to relevant journals and offer national and international presentations. This study will enable better characterisation of the impact of the stay-at-home (related to COVID-19 pandemic) on the mental health of children and adolescents.

Trial registration number NCT04498416.

\section{INTRODUCTION}

In the context of a viral outbreak and the stay-at-home measures, a significant increase in psychiatric disorders has previously been reported in the adult population. ${ }^{1}$

Large Chinese studies involving 52730 participants aged 18 and more, recently reported that following COVID-19 pandemic and stay-at-home, $35 \%$ of the participants exhibit moderate stress while $5.14 \%$ are characterised by severe stress. ${ }^{2}$ Furthermore, adults exhibit fear (20\%), nervousness $(18 \%)$, sadness $(18 \%)$ and guilt $(10 \%) .^{3}$

Up to today, there is a lack of data in paediatric population. Psychiatric disorders that may result from stay-at-home, in paediatric population, in the context of coronavirus outbreak include post-traumatic stress disorder (PTSD), 
anxiety, major depressive disorder and emotional dysregulation. ${ }^{4-7}$ Another study found that COVID-19 pandemic and stay-at-home lead to anxiety, 22\% insomnia, 13\% symptoms of depression and $13 \%$ somatic complaints for Wuhan's children. ${ }^{8}$ Pierce et al conducted a cohort study on the effects of stay-at-home on mental health. ${ }^{9}$ Their results demonstrate a deterioration of mental health in 2020, compared with 2018. The populations most at risk of presenting difficulties are, in their study, adolescents, women, parents of preschool children and people with economic difficulties before the stay-at-home. ${ }^{9}$

In addition, specific stress factors related to the stay-athome such as the loss of habitual routine, ${ }^{10}$ the reduction of social contact ${ }^{11}$ or the increase in addictions (eg, video games, television, cannabis, tobacco, alcohol) ${ }^{12} 13$ can lead to psychiatric disorders in the paediatric population. ${ }^{4}$ Fear of being infected or to transmit infection, unusual bereavement, return to 'normal life' with its anterior fear (separation anxiety, school phobia, social anxiety, etc), ${ }^{14}$ exposition to media reporting stressful news, may be added to previous stress factors. ${ }^{15} 16$ In a literature review, Ye explains that certain symptoms can be common in children (trouble sleeping and decreased appetite). ${ }^{17}$ Other symptoms may be specific to developmental periods. Thus, preschool children are at risk of developing crying and becoming clingier. School children can be more nervous and scared. Finally, adolescents can be worried, irritable or angry. ${ }^{17}$

We hypothesise that the present COVID-19 pandemic and stay-at-home could, thus, represent a traumatic event for a large number of people, including children and adolescents. Currently, several researchers note the current limits on psychiatric diagnoses, in particular, concerning PTSD. For example, Horesh and Brown propose to understand the status of this crisis as a traumatic event. ${ }^{18}$

For several clinicians, these experiences might evolve to include a broad range of 'public mental health concerns', including distress reactions (insomnia, anger, extreme fear) and psychiatric disorders (PTSD, anxiety disorders, depression, somatisation). ${ }^{19}$ Exceptional epidemic situations also promoted PTSD in the past. ${ }^{20}$ Considering that humanity is undergoing the most severe pandemic since Spanish Influenza, the actual pandemic of COVID-19 is very likely to promote PTSD. ${ }^{21}$

Children and adolescents exposed to a traumatic event can mainly develop PTSD. According to the Diagnostic and Statistical Manual of Mental Disorders, Fifth Edition (DSM-5) classification, PTSD is a chronic disorder characterised by four cardinal groups of symptoms: intrusive memories, avoidance behaviours, cognitive and mood disturbances and neurovegetative overactivation. ${ }^{22}$ The symptomatology in paediatric population might be expressed differently, with repetitive games on the theme of contagion, sleep disturbances with difficulty falling asleep and nightmares, avoidance of the information related to COVID-19, anger bursts and unusual sadness. $^{1323}$
The incidence of PTSD in children and adolescents after a traumatic exposure is estimated to $15.8 \% .{ }^{24}$ Epidemiological studies conducted on the consequences of traumatic exposure reveal a high variability in the development of psychiatric disorders. Several factors may explain the heterogeneity of the results, including age of exposure, type of trauma experienced, change in individual's fundamental beliefs (Beliefs in a Just World) or a previous history of anxiety disorders or previous exposure to traumatic event. ${ }^{25}$ PTSD in children is considered as a risk factor for many psychiatric disorders. It is estimated that $75 \%$ of PTSD children and adolescents develop comorbidities. ${ }^{26}$ Among these disorders, the most frequent are anxiety disorders, attention deficit hyperactivity disorder and depression in paediatric populations. ${ }^{27} 28$

As in adult population, early intervention is recommended for child and adolescent during crisis (American Academy of Child and Adolescent Psychiatry: AACAP) ${ }^{29}$ The aim of these interventions is to reduce the risk of developing PTSD or clinical manifestations. It is also important to take into account the protective aspects such as family, school or friends.

AACAP $^{30}$ recently provided specific recommendations for COVID-19 pandemic and stay-at-home: trying to create a space for the child to speak where the adult answers questions with honesty, with a vocabulary adapted to the child's age, recognise and accept the child's thoughts, emotions and reactions, being reassuring without making any unachievable promises and avoiding exposure to overly frightening information. For practitioners, it is important to pay special attention to children and adolescents with a history of serious illness, bereavement or traumatic exposure. ${ }^{31}$

The main goal of Coronavirus Confinement 2020 (CoCo20) study is to assess prospectively the impact of the coronavirus pandemic (COVID-19) and the stay-athome on the development of psychiatric disorders in children and adolescent.

\section{METHODS AND ANALYSIS Objectives}

The main objective of this study is to assess the development of psychiatric disorders (including PTSD) in child and adolescent outpatients during and after the stay-athome related to COVID-19 pandemic. The secondary objectives are to highlight the effect of prior traumatic experiences, anterior psychiatric disorders and parental stress reactions on the development of psychiatric disorders (during and after the stay-at-home related to COVID-19 pandemic).

\section{Trial design}

CoCo20 is a longitudinal and multicentric research (Nice, Marseille and Avignon) involving human beings. Risks and constraints associated with this study are minimal. No health products were used. The recruiting centres belong to the Regional Psychotrauma Centre 


\begin{tabular}{|c|c|c|c|}
\hline & Vo & V1 and V3 & V2 and V4 \\
\hline Inclusion criteria & $x$ & & \\
\hline Informed consent & $x$ & & \\
\hline Emergency semi-directed interview & $x$ & $x$ & \\
\hline $\mathrm{DIPA}_{1} / \mathrm{KSADS} 2$ & & & $x$ \\
\hline Conners & & $\mathrm{x}$ & $\mathrm{x}$ \\
\hline WFIRS3 & & $x$ & \\
\hline $\mathrm{PSI}_{-} \mathrm{SF}_{4}$ & & $\mathrm{X}$ & \\
\hline $\mathrm{YCPC}_{5} / \mathrm{CPC}_{6} / \mathrm{PCL}_{7}$ & & & $x$ \\
\hline PAS8 / STAIC9 / STAl10 & & & $x$ \\
\hline PHQ-13 11 & & $x$ & \\
\hline
\end{tabular}

Figure 1 'CoCo 20' procedure and evaluation criteria for the first study times. DIPA, Diagnostic Infant preschool Assessment; KSADS, Kiddie Schedule for Affective Disorders and Schizophrenia; WFIRS, Weiss functional impairment rating scale; PSI-SF, Parental stress index-short form; YCPC, Young Child PTSD Checklist; CPC, Child PTSD Checklist; PCL, PTSD Checklist; PAS, Preschool anxiety scale; For 10 peer review only-http://bmjopen.bmj.com/site/about/guidelines.xhtml; STAIC, Situational Anxiety and Trait Inventory for Child; STAI, Situational Anxiety and Trait Inventory; PHQ-13, Patient Health Questionnaire-13.

PACA Corse (France), based at Cote d'Azur University, including Avignon, Marseille and Nice centres. It is an ongoing study with the inclusion period starting in March 2020 and ending in November 2020.

This study is offered to children who use one of the recruiting centres during stay-at-home or at the end of the stay-at-home, either by appointment requests or by the special emergency COVID-19 hotline. Children and adolescents may be enrolled during stay-at-home (five visits) or after stay-at-home (three visits).

During the stay-at-home, participants will be assessed at baseline (V0), 1 week after baseline (V1), 1 month after baseline (V2), 1 week after the end of the stay-at-home (V3) and 1 month after the end of the stay-at-home (V4). The expected duration of the assessment varies according to the length of the visit: 30 min (V0), 1 hour (V1 and V3) and 1 hour $30 \mathrm{~min}$ (V2 and V4).

After the stay-at-home, only the first three visits are done (V0, V3 and V4). During the stay-at-home, the visits are realised by phone and/or visioconsultation. After the end of the stay-at-home, V3 and V4 will be performed face-to-face. Measures administered are summarised in figure 1 . The investigators carrying out the assessments will be trained in paediatric psychotrauma.

Participants: to compare the difference between our population of participants confined following the COVID-19 epidemic and the general population (European Study of the Epidemiology of Mental Disorders, ESEMeD),${ }^{32}$ our criterion of primary judgement is the prevalence of PTSD.

We estimate a sample size of 70 participants, considering an alpha of 0.05 , a statistical power of $80 \%$ and an increase of $15 \%$ experimental loss. If the recruitment of 70 people is reached, we will continue the inclusions to improve statistical power and meet the secondary assumptions.

To perform the secondary objectives, we defined three groups:

- Group 1: children and adolescents with a prior traumatic experience.

- Group 2: children and adolescents with a history of psychiatric disorder (DSM 5) except exclusion criteria.

- Group 3: children and adolescents without a history of psychiatric disorders (DSM 5) and without follow-up care, recruited among people who call emergency COVID-19 hotline.

\section{Inclusion criteria}

This study is proposed to each child and adolescent outpatient consulting during COVID-19 pandemic and stay-at-home in medical-psychological centres (Marseille and Avignon), the Paediatric Psychotrauma Regional Centre of Nice or who call emergency COVID-19 hotline:

- Age of 0-18years.

- Affiliated with a social security scheme.

- Fluent in French.

- Children whose parents have accepted participation in the study (collection of informed consents).

\section{Noninclusion criteria}

- Children and adolescents with medical conditions hindering the comprehension of the study goals and flow (severe communication and/or cognitive disability and psychiatric emergencies).

- Persons deprived of liberty by judicial or administrative decision. 
- Persons subjected to a period of exclusion for another research.

- A participant may be discontinued from the study at any time if the children or his parent withdraws consent.

\section{Measures}

Quantitative and qualitative assessments are administered to the participants (summarised in figure 1):

- Emergency semistructured interview: this is a semistructured interview conducted in two stages: at the time of the trauma and a few days later. This identifies immediate and delayed symptoms of stress response in children, depending on their age. This interview was developed by the Paediatric Psychotrauma Regional Centre of Nice team and authorised by the Regional Health Agency (ARS Provence Alpes Côte d'Azur).

- Diagnosis Infant and Preschool Assessment (DIPA): is a semistructured psychiatric interview with parents of children under the age of $7 .^{33}$ DIPA assesses the occurrence, frequency and intensity of children's symptoms through parental observation.

- Kiddie Schedule for Affective Disorders and Schizophrenia (K-SADS): is a semistructured interview designed for children aged 7 years or older. ${ }^{35}$ It involves both an interview with the parent and one with the child, and it covers both current issues (including the assessment of current psychiatric disorder) as well as prior episodes of the disorder.

- Conners Scale-short version: this questionnaire collects and quantifies the symptoms of Attention Deficit Disorders with or without Hyperactivity Disorder, ${ }^{37}$ for children aged 6-18.

- Weiss functional impairment rating scale-parental version $^{38}$ assesses the emotional and behavioural skills of individuals. The parent completes this scale.

- Parenting stress index-short form ${ }^{39}$ measures the extent of parental stress, parent-child relationship and parents' difficulties in the day-to-day management of children.

- PTSD Scales: the frequency and intensity of PTSD symptoms will be assessed using the Young Child PTSD Checklist-YCPC ${ }^{41}$ for children aged 3 months to 6 years and 11 months, the CPC (Child PTSD checklist) ${ }^{42}$ for children aged 7 years to 17 years and 11 months and with the PCL 5 (PTSD checklist) ${ }^{44} 45$ for adults.

- Anxiety Scale: the intensity of anxiety will also be objectified using the Preschool Anxiety Scale ${ }^{46}$ for children aged 3 months to 5 years and 11 months. For children aged 6 years or more, anxiety will be assessed through the Situational Anxiety and Child Trait Inventory (STAI-C). ${ }^{47}$ For adult participants, we will use STAI.

- Patient Health Questionnaire(PHQ) 13: is a questionnaire that assesses physical symptoms in children and adolescents aged 6-17 years. It is adapted from PHQ-15, a 15-item self-report scale (DSM5-level 2-somatic symptom-parent/guardian). ${ }^{48}$ Participants need to evaluate the extent of the somatic symptoms on a 3-point scale ranging from 'not bothered at all' (value $=0$ ) to 'bothered a lot' (value $=2$ ).

For children under 6 years old, interviews are realised with the child's parents/caregivers. They are the ones who answer the interviews and fill out the questionnaires about children's behaviours and symptoms.

For children aged 6-12, interviews are carried out either with the child only or in the presence of the child and the parent if needed by the child (eg, if this makes the child feel safer). The psychologist reformulates the questions until the child understands them.

For adolescents, the interview is done directly with them, then with the parents'.

\section{Data collection process}

Each investigator at the different study centres (medicalpsychological centres (Marseille and Avignon), the Paediatric Psychotrauma Regional Centre of Nice or who call emergency COVID-19 hotline) is responsible for the collection and the data completion on the Case Report Form after the assessment . Statistical analysis will be performed by The Child and Adolescent Psychiatry University Unit (Service Universitaire de Psychiatrie de l'Enfant et de l'Adolescent SUPEA). The R software will be used to perform statistical analysis.

\section{Statistical analysis}

Descriptive statistics (mean, mode and SD) will be carried out on all the data obtained, and then according to the three experimental groups. Main component analyses and factor analyses will also be performed.

To test the impact of prior traumatic exposure in the development of psychiatric disorder during COVID-19 pandemic and consecutive stay-at-home, we will perform student t-test to compare the group 1 (PTSD) and group 3 (healthy). To test the impact of anterior history of psychiatric disorders in the development of current psychiatric disorder during COVID-19 pandemic and consecutive stay-at-home, we will perform student t-test to compare the group 2 (previous history of psychiatric disorders) and group 3 (healthy).

Step-by-step regressions and correlations (BravaisPearsons) will be made between clinical data, sociodemographic data and psychiatric (K-SADS, DIPA, CPC and YCPC) test scores of subjects. Step-by-step regressions and correlations (Bravais-Pearsons) will also be performed between clinical evaluations of parents and those of the child. Level of significance for the $\mathrm{p}$ value was fixed at $<0.05$.

\section{Outcomes and implications}

This study will provide for the child/ adolescent and his family:

- An early diagnosis of possible psychological distress or psychiatric disorder related to the pandemic and the 
stay-at-home, allowing a rapid implementation of an appropriate care.

- A consideration of their immediate difficulties that often has immediate benefits on the quality of life and can help to better live stay-at-home and the end of it.

- A dedicated time for the child or adolescent to speak and share his feelings about the stay-at-home. A designated therapist will be assigned to each patient on entry, allowing him/her to share the potential difficulties more easily over the five or three sessions.

- For the community, the study will provide documented scientific knowledge in the field of paediatric psychotrauma in pandemic/stay-at-home context.

\section{Patient and public involvement}

Results will systematically be transmitted to patients and legal caregivers. Results will also be discussed with the referent psychiatrist of child psychiatry care if existing.

\section{DISCUSSION}

Both the current pandemic and the stay-at-home environment raise a high level of distress and fear for children. ${ }^{4}$

Exceptional epidemic situations are known to promote psychiatric disorders, including PTSD. ${ }^{18}$ Considering that humanity is undergoing the most severe pandemic since Spanish nfluenza, the actual pandemic of COVID-19 is very likely to lead to psychiatric difficulties. ${ }^{21}$ During the stay-at-home, these difficulties can be explained by several factors including the change in lifestyle, ${ }^{49} 50$ through reduced social interaction ${ }^{11}$ and repeated exposure to media and news. ${ }^{12} 16$ The closure of schools, lack of outdoor activity, aberrant eating and sleeping routines are likely to disrupt the usual way of life of children and can potentially promote monotony, distress, impatience, embarrassment and various psychiatric manifestations. ${ }^{10}$

In addition to stay-at-home measures, studies carried out on previous health crises (H1N1, Ebola, etc) indicate an increase in acts of violence and cumulative trauma. It has been found, for example, that incidents of domestic violence and child abuse have been increasing since the confinement. ${ }^{100}$ Cohen and Bosk ${ }^{51}$ discuss the financial difficulties; not only stress and anxiety associated with the pandemic, potential negative family interactions but also limited access to health and legal resources. Indeed, many services (child protection, educators and social workers) have reduced home visits and mediatised visits, due to the COVID-19. In a similar way, the closure of schools also isolated children. ${ }^{51}$

Children with a history of psychiatric disorder, especially those with history of traumatic exposure,$^{50}$ may present increased distress. ${ }^{52} 53$ These difficulties can take different forms, according to the child's age. ${ }^{13}{ }^{54}$ In younger children, sleep disturbances, separation anxiety or psychomotor agitation may occur. ${ }^{55}$ In school-aged children, cognitive impairments, anxiety attacks or obsessivecompulsive behaviours have previously been reported. ${ }^{56}$ Among adolescents, the literature also warns us about the risk of developing addictions (eg, video games), opposition or risk behaviours. ${ }^{57} 58$ These different responses may be accentuated by previous exposure to traumatic events. ${ }^{1331}$

In addition, parental distress is also known as a factor that can increase psychiatric disorders in their children. Faced with a crisis situation, children are essentially dependent on referent adults (parents, guardians, teachers etc) to determine their emotional and behavioural responses. It has, thus, been shown that when the 'referent figures' manage to secure the child and explain the situation to him, child presents a better level of psychological well-being. ${ }^{59}$

Moreover, the distress of the parents and/or guardians of the child during the health crisis and the stay-at-home was correlated with the increase in psychological and psychiatric problems of young children. ${ }^{60}$ Many parents have to face financial difficulties related to the current pandemic or are extremely stressed by health measures at work, in particular, teleworking. ${ }^{14}$ Studies of past pandemics (eg, H1N1 epidemic) show that the cumulative effects of parental stress and fear of becoming ill increase the risk of vicarious trauma in children. ${ }^{61}$

The consequences of health crisis in paediatric population are currently not well documented. A better knowledge of consequences of stay-at-home may lead to the development of prevention guidelines and professional practices. These protocols may thus be used whatever the cause of the stay-at-home (eg, chemical leaks, terrorist attack, pandemic) in order to react rapidly and adapt the specific care to promote paediatric resilience.

Resilience emerges from ordinary adaptive systems such as close relationships with competent and caring adults and peers, effective schools and communities, opportunities to succeed and beliefs in the self. ${ }^{62}$ Clinical services should offer preventative support and early intervention where possible and be prepared for an increase in mental health problems. ${ }^{63}$

\section{Perspectives}

The interest of CoCo20 is to deepen the knowledge in the field of paediatric psychotrauma population, during pandemic crisis. The current health crisis affects children and adolescents in different areas: clinical, developmental, social, etc. The clinical care offered to these populations must be adapted to this context and to the suffering of these young people.

\section{Limitations of the study}

The main limitations of the CoCo20 protocol are the nonrepresentability of the entire population and attrition.

The population of the Nice region due to its recent history (attack of 14 July 2016) has already been made aware of psychotrauma and its clinical manifestations, this factor should be taken into account in statistical analyses. Similarly, experimental loss in longitudinal study is known. This phenomenon can generate a loss of statistical power, selection biases or erroneous conclusions. To 
prevent attrition, all participants are contacted by phone on a regular basis. During the stay-at-home, the frequency of calls was weekly. At the end of the stay-at-home, for children needing reassurance, face-to-face appointments were made, and then phone news was taken every 15 days

\section{ETHICS AND DISSEMINATION}

The CoCo20 protocol was approved by the Ethics Committee of Cote d'Azur University (CERNI) (number 2020-59). All patients and their legal caregivers signed a written informed consent on enrolment in the study. Inclusions started in March 2020.

We will submit the results of the study to relevant journals and offer national and international presentations. This study will enable better characterisation of the impact of the stay-at-home (related to COVID-19 pandemic) on the mental health of children and adolescents.

\section{Author affiliations}

${ }^{1}$ Service Universitaire de Psychiatrie de l'Enfant et de l'Adolescent (SUPEA), Hôpitaux Pédiatriques de Nice CHU-Lenval (HPNCL), Nice, Provence-Alpes-Côte d'Azur, France

${ }^{2}$ CoBTek, FRIS, Université Côte d'Azur, Nice, Provence-Alpes-Côte d'Azur, France

Acknowledgements The authors are grateful to Manon Lucibello (Psychologue, Centre Hospitalier Montfavet, Avignon), Dr Olivier Fossard (Psychiatre, Centre Hospitalier Montfavet, Avignon), Pr Francois Poinso (Pédopsychiatre, Assistance Publique Hôpitaux de Marseille, CHU, Sainte Marguerite) and Dr Jokthan Guivarch (Pédopsychiatre, Assistance Publique Hôpitaux de Marseille, CHU, Sainte Marguerite) for their contribution.The authors are grateful to Floriane Vallée and Léa Bruni for their work.The authors are grateful to Anamaria Bogdan and Maria Capovilla for revising the manuscript.

Contributors MG, AF, AR, ON, MB, FA contributed to the conceptualisation of the CoCo20 study and preparation of grant application. MG, AF, AR, ON, MB, FA are involved in clinical assessments of the patients. All authors are involved in the interpretation of results. MG and $\mathrm{AF}$ have been involved in drafting the first version of the manuscript, and all authors have revised it critically for important intellectual content, and approved the final version.

Funding The authors have not declared a specific grant for this research from any funding agency in the public, commercial or not-for-profit sectors.

Competing interests None declared.

Patient and public involvement Patients and/or the public were not involved in the design, or conduct, or reporting, or dissemination plans of this research.

Patient consent for publication Not required.

Provenance and peer review Not commissioned; externally peer reviewed.

Open access This is an open access article distributed in accordance with the Creative Commons Attribution Non Commercial (CC BY-NC 4.0) license, which permits others to distribute, remix, adapt, build upon this work non-commercially, and license their derivative works on different terms, provided the original work is properly cited, appropriate credit is given, any changes made indicated, and the use is non-commercial. See: http://creativecommons.org/licenses/by-nc/4.0/.

ORCID iD

Arnaud Fernandez http://orcid.org/0000-0002-6326-9096

\section{REFERENCES}

1 Hawryluck L, Gold WL, Robinson S, et al. Sars control and psychological effects of quarantine, Toronto, Canada. Emerg Infect Dis 2004;10:1206-12.

2 Qiu J, Shen B, Zhao M, et al. A nationwide survey of psychological distress among Chinese people in the COVID-19 epidemic: implications and policy recommendations. Gen Psychiatr 2020;33:e100213.

3 Reynolds DL, Garay JR, Deamond SL, et al. Understanding, compliance and psychological impact of the SARS quarantine experience. Epidemiol Infect 2008;136:997-1007.

4 Brooks SK, Webster RK, Smith LE, et al. The psychological impact of quarantine and how to reduce it: rapid review of the evidence. Lancet 2020;395:912-20.

5 Duan L, Shao X, Wang Y, et al. An investigation of mental health status of children and adolescents in China during the outbreak of COVID-19. J Affect Disord 2020;275:112-8.

6 Zhou SJ, Zhang LG, Wang LL. Prevalence and socio-demographic correlates of psychological health problems in Chinese adolescents during the outbreak of COVID-19. Eur Child Adolesc Psychiatry 2020:1-10.

7 Gindt M, Fernandez A, Battista M, et al. [Psychiatric consequences of Covid 19 pandemic in the pediatric population]. Neuropsychiatr Enfance Adolesc 2021. doi:10.1016/j.neurenf.2021.01.001. [Epub ahead of print: 0901 2021]

8 Cui Y, Li Y, Zheng Y. Cs of child. mental health services for children in China during the COVID-19 pandemic: results of an expert-based national survey among child and adolescent psychiatric hospitals. Eur Child Adolesc Psychiatry 2020;1.

9 Pierce M, Hope H, Ford T, et al. Mental health before and during the COVID-19 pandemic: a longitudinal probability sample survey of the UK population. Lancet Psychiatry 2020;7:883-92.

10 Ghosh R, Dubey MJ, Chatterjee S, et al. Impact of COVID -19 on children: special focus on the psychosocial aspect. Minerva Pediatr 2020;72:34

11 Witt A, Ordóñez A, Martin A. Child and adolescent mental health service provision and research during the Covid-19 pandemic: challenges, opportunities, and a call for submissions, 2020.

12 Chen I-H, Chen C-Y, Pakpour AH, et al. Internet-related behaviors and psychological distress among schoolchildren during COVID-19 school suspension. J Am Acad Child Adolesc Psychiatry 2020;59:1099-1102.e1:1099-102.

13 Imran N, Zeshan M, Pervaiz Z. Mental health considerations for children \& adolescents in COVID-19 Pandemic. Pak J Med Sci 2020;36:S4.

14 Wade M, Prime H, Browne DT. Why we need longitudinal mental health research with children and youth during (and after) the COVID-19 pandemic. Psychiatry Res 2020;290:113143.

15 Garfin DR, Silver RC, Holman EA. The novel coronavirus (COVID-2019) outbreak: amplification of public health consequences by media exposure. Health Psychol 2020;39:355-7.

16 Rapa E, Dalton L, Stein A. Talking to children about illness and death of a loved one during the COVID-19 pandemic. Lancet Child Adolesc Health 2020;4:560-2.

$17 \mathrm{Ye} \mathrm{J}$. Pediatric mental and behavioral health in the period of quarantine and social distancing with COVID-19. JMIR Pediatr Parent 2020;3:e19867.

18 Horesh D, Brown AD. Traumatic stress in the age of COVID-19: a call to close critical gaps and adapt to new realities. Psychol Trauma 2020;12:331-5.

19 Shigemura J, Ursano RJ, Morganstein JC, et al. Public responses to the novel 2019 coronavirus (2019-nCoV) in Japan: mental health consequences and target populations. Psychiatry Clin Neurosci 2020;74:281-2

20 Imran N, Aamer I, Sharif MI, et al. Psychological burden of quarantine in children and adolescents: a rapid systematic review and proposed solutions. Pak J Med Sci 2020;36:1106.

21 Dutheil F, Mondillon L, Navel V. PTSD as the second tsunami of the SARS-Cov-2 pandemic. Psychol Med 2020:1-2.

22 American Psychiatric Association. Diagnostic and statistical manual of mental Disorder-5, 2013.

23 Dyregrov A, Yule W. A review of PTSD in children. Child Adolesc Ment Health 2006;11:176-84.

24 Alisic E, Zalta AK, van Wesel F, et al. Rates of post-traumatic stress disorder in trauma-exposed children and adolescents: meta-analysis. Br J Psychiatry 2014;204:335-40.

25 Trickey D, Siddaway AP, Meiser-Stedman R, et al. A meta-analysis of risk factors for post-traumatic stress disorder in children and adolescents. Clin Psychol Rev 2012;32:122-38.

26 Bouvard M. Échelles et Questionnaires d'évaluation Chez l'enfant et l'adolescent. Elsevier Masson, 2008.

27 Famularo R, Fenton T, Kinscherff R, et al. Psychiatric comorbidity in childhood post traumatic stress disorder. Child Abuse Negl 1996;20:953-61.

28 Kar N, Bastia B. "Post-Traumatic Stress Disorder, Depression and Generalized Anxiety Disorder in Adolescents after a Natural Disaster: A Study of Comorbidity.". Clin Pract Epidemiol Ment Health 2006;1:17. 
29 Cohen JA, Bukstein O, Walter $\mathrm{H}$, et al. Practice parameter for the assessment and treatment of children and adolescents with posttraumatic stress disorder. J Am Acad Child Adolesc Psychiatry 2010;49:414-30.

30 AACAP. "Talking to Children about Coronavirus (Covid 19).", 2020. Available: https://www.aacap.org/App_Themes/AACAP/Docs/latest_ news/2020/Coronavirus COVID19 Children.pdf

31 Stark AM, White AE, Rotter NS, et al. Shifting from survival to supporting resilience in children and families in the COVID-19 pandemic: lessons for informing U.S. mental health priorities. Psychol Trauma 2020;12:S133-5.

32 Lépine J-P, Gasquet I, Kovess V, et al. Prévalence et comorbidité des troubles psychiatriques dans la population générale française : résultats de l'étude épidémiologique ESEMeD/MHEDEA 2000/ (ESEMeD). Encephale 2005;31:182-94.

33 Scheeringa MS. Untangling psychiatric comorbidity in young children who experienced single, repeated, or Hurricane Katrina traumatic events. Child Youth Care Forum 2015;44:475-92.

34 Thümmler S, Gindt M, Battista M. Diagnostic infant and preschool assessment (DIPA) (French version 19/07/17 of DIPA DSM-5 Scheeringa 08/08/15, 2017.

35 Kaufman J, Birmaher B, Axelson D. The schedule for affective disorders and schizophrenia for school-age children-present and lifetime version for DSM5 (K-SADS-PL-DSM5. Child and Adolescent Research and Education (CARE) Program. Western Psychiatric Institute and Clinic: Advanced Center for Intervention and Services Research (ACISR) for Early Onset Mood and Anxiety Disorders, Yale University, 2016.

36 Thümmler S, Askenazy F. K-SADS-PL DSM-5 French version Mai 2018 (of K-SADS-PL DSM-5 November 2016, Kaufmann J, Birmaher B, Axelson D, Perepletchikova F, Brent D, Ryan N, 2018.

37 Egeland J, Kovalik-Gran I. Validity of the factor structure of Conners' CPT. J Atten Disord 2010;13:347-57.

38 Thompson T, Lloyd A, Joseph A, et al. The Weiss functional impairment rating Scale-Parent form for assessing ADHD: evaluating diagnostic accuracy and determining optimal thresholds using ROC analysis. Qual Life Res 2017;26:1879-85.

39 Abidin R. Short Form: Professional Manual." Parenting Stress Index. Third ed. Odessa, FL: Psychological Assessment Resources, Inc, 1995.

40 Scheeringa M. Young child PTSD checklist, 2013.

41 Thümmler S, Gindt M, Maria F. Young child PTSD checklist (YCPC) (French version 18/05/17 of YCPC DSM-5, Scheeringa 23/05/14), 2017. Available: http://medicine.tulane.edu/departments/psychiatry/ research/dr-scheeringas-lab/manuals-measures-trainings

42 Scheeringa M. Child PTSD checklist - parent version (CPC-P). Tulane University, 2014.

43 Thümmler S, Gindt M, Maria F. Child PTSD checklist (CPC) (French version 18/05/17 of CPC DSM-5, Scheeringa), 2017. Available: http://medicine.tulane.edu/departments/ psychiatry/research/drscheeringas-lab/manuals-measures-trainings

44 Weathers FW, Litz BT, Keane TM. The ptsd checklist for dsm-5 (pcl-5). In: Scale available from the National center for PTSD, 2013. https://www.ptsd.va.gov/

45 Ashbaugh AR, Houle-Johnson S, Herbert C, et al. Psychometric validation of the English and French versions of the posttraumatic stress disorder checklist for DSM-5 (PCL-5). PLoS One 2016;11:e0161645

46 Spence SH, Rapee R, McDonald C, et al. The structure of anxiety symptoms among preschoolers. Behav Res Ther 2001;39:1293-316.

47 Spielberger CD. State-Trait anxiety inventory. In: The Corsini encyclopedia of psychology, 2010

48 Kroenke K, Spitzer RL, Williams JBW. The PHQ-15: validity of a new measure for evaluating the severity of somatic symptoms. Psychosom Med 2002;64:258-66.

49 Bahn GH. Coronavirus disease 2019, school closures, and children's mental health. Soa Chongsonyon Chongsin Uihak 2020;31:74-9.

50 Courtney D, Watson P, Battaglia M, et al. COVID-19 impacts on child and youth anxiety and depression: challenges and opportunities. Can J Psychiatry 2020;65:688-91.

51 Cohen RIS, Bosk EA. Vulnerable youth and the COVID-19 pandemic. Pediatr 2020;146.

52 Amstadter AB, Elwood LS, Begle AM, et al. Predictors of physical assault victimization: findings from the National survey of adolescents. Addict Behav 2011;36:814-20.

53 Hodges M, Godbout N, Briere J, et al. Cumulative trauma and symptom complexity in children: a path analysis. Child Abuse Negl 2013;37:891-8.

54 Bomyea J, Lang AJ. Emerging interventions for PTSD: future directions for clinical care and research. Neuropharmacology 2012;62:607-16.

55 Salmon K, Bryant RA. Posttraumatic stress disorder in children. The influence of developmental factors. Clin Psychol Rev 2002;22:163-88.

56 Thabet AA, Vostanis P. Post traumatic stress disorder reactions in children of war: a longitudinal study. Child Abuse Negl 2000;24:291-8

57 Cisler JM, Amstadter AB, Begle AM, et al. PTSD symptoms, potentially traumatic event exposure, and binge drinking: a prospective study with a national sample of adolescents. J Anxiety Disord 2011;25:978-87.

58 Fegert J, Freyberger HJ. Posttraumatische Belastungsstörungen (PTBS) UPTBS und Traumafolgestörungen in der Adoleszenz [Posttraumatic stress disorders (PTSD) and trauma sequel disorders in adolescence]. Fortschr Neurol Psychiatr 2019;87:638-41.

59 Diab M, Peltonen K, Qouta SR, et al. Can functional emotion regulation protect children's mental health from war trauma? A Palestinian study. Int J Psychol 2019;54:42-52.

60 Gurwitch RH, Salem H, Nelson MM, et al. Leveraging parent-child interaction therapy and telehealth capacities to address the unique needs of young children during the COVID-19 public health crisis. Psychol Trauma 2020;12:S82-4.

61 Steele W. Trauma in schools and communities: recovery lessons from survivors and responders. Routledge, 2015.

62 Dvorsky MR, Breaux R, Becker SP. Finding ordinary magic in extraordinary times: child and adolescent resilience during the COVID-19 pandemic. Eur Child Adolesc Psychiatry 2020:1-3.

63 Loades ME, Chatburn E, Higson-Sweeney N, et al. Rapid systematic review: the impact of social isolation and loneliness on the mental health of children and adolescents in the context of COVID-19. J Am Acad Child Adolesc Psychiatry 2020;59:1218-39. 